\title{
Gamma-rays signature of dark matter in the CTA era: status and prospects
}

\author{
Aldo Morselli* \\ INFN Roma Tor Vergata, Italy \\ E-mail: aldo.morselieroma2.infn.it
}

High-energy phenomena in the cosmos, and in particular processes leading to the emission of gamma- rays in the energy range $10 \mathrm{MeV}-100 \mathrm{TeV}$, play a very special role in the understanding of our Universe. This energy range is indeed associated with non-thermal phenomena and challenging particle acceleration processes. The Universe can be thought as a context where fundamental physics, relativistic processes, strong gravity regimes, and plasma instabilities can be explored in a way that is not possible to reproduce in our laboratories. High-energy astrophysics and atmospheric plasma physics are indeed not esoteric subjects, but are strongly linked with our daily life. Understanding cosmic high-energy processes has a large impact on our theories and laboratories applications. The technology involved in detecting gamma-rays is challenging and drives our ability to develop improved instruments for a large variety of applications [1].

In the last decades a vast amount of evidence for the existence of dark matter has been accumulated. At the same time, many efforts have been undertaken to try to identify what dark matter is. Indirect searches look at places in the Universe where dark matter is believed to be abundant and seek for possible annihilation or decay signatures.

At high energies, the Cherenkov Telescope Array (CTA) represents the next generation of imaging Cherenkov telescopes and, with one site in the Southern hemisphere and one in the Northern hemisphere, will be able to observe all the sky with unprecedented sensitivity and angular resolution above a few tens of GeV. The CTA Consortium will undertake an ambitious program of indirect dark matter searches for which we report here the brightest prospects.

At lower energies, the energy range between 1 and $100 \mathrm{MeV}$ is an experimentally very difficult range and remained uncovered since the time of COMPTEL. New instruments can address all astrophysical issues left open by the current generation of instruments. In particular, a good angular resolution in the energy range $10 \mathrm{MeV}-1 \mathrm{GeV}$ is crucial to resolve patchy and complex features of diffuse sources in the Galaxy and in the Galactic Centre as well as increasing the point source sensitivity. This instrument addresses scientific topics of great interest to the community, with particular emphasis on multifrequency correlation studies involving radio, optical, IR, X-ray, soft gamma-ray and $\mathrm{TeV}$ emission. The possibility to study not only the pair production regime but also the Compton regime with this kind of detectors is currently under investigation and it is another possible very interesting breaktrough.

Frontier Research in Astrophysics-III

28 May - 2 June 2018

Palermo, Italy

${ }^{*}$ Speaker. 


\section{The high enery frontier}

The existence of dark matter (DM) in our Universe is well established, but its nature is at present still unknown. Multiple hypotheses endure as to the character of dark matter and for the most popular models discussed in literature CTA has a unique chance of discovery. Among the most promising particle candidates are Weakly Interacting Massive Particles (WIMPs), which typically can self-annihilate to produce prompt or secondary gamma-rays during the annihilation. If WIMPs are produced thermally in the early Universe then the current velocity-averaged self-annihilation cross-section has a natural value of approximately $\left\langle\sigma_{a n n} v\right\rangle \sim 3 \cdot 10^{-26} \mathrm{~cm}^{3} \mathrm{~s}^{-1}$ [2]. WIMPs models, such as the supersymmetric neutralino, give predictions for gamma-ray energy spectra from the annihilations, which are crucial inputs, together with the DM distribution in the observed target, to estimate prospects for the sensitivity of indirect searches. The goal of the present study is to provide preliminary comparative expectations on indirect DM searches with the Cherenkov Telescope Array (CTA) $[4,5]$ taking into account continuum gamma-ray signatures coming from typical DM annihilation channels. The expected DM annihilation gamma-ray flux from a DM-dominated region depends on the so-called particle physics and astrophysical (or $J$ ) factors:

$$
\Phi_{s}(\Delta \Omega)=\frac{1}{4 \pi} \frac{\langle\sigma v\rangle}{2 m_{D M}^{2}} \int_{E_{\min }}^{E_{\max }} \frac{d N_{\gamma}}{d E_{\gamma}} d E_{\gamma} \times J(\Delta \Omega)
$$

where $\langle\sigma v\rangle$ is the velocity-averaged self-annihilation cross-section, $m_{D M}$ is the dark matter particle mass, $E_{\min }$ and $E_{\max }$ are the energy limits for the measurement and $\frac{d N_{\gamma}}{d E_{\gamma}}$ is the energy spectrum of the gammas produced in the annihilation (as, e.g., from [6]). The products of DM annihilation are thought to come from decay and/or hadronization of the primary Standard Model (SM) particles: quark-antiquark, lepton and boson, and each channel is expected to have its own branching ratio. The $J$ factor is the integral along the line of sight of the squared DM density profile of the given target integrated within an aperture angle, $\int_{\Delta \Omega} \mathrm{d} \Omega \int_{\text {l.o.s. }} \rho_{D M}^{2}(r) \mathrm{d} l$. Until recently, simulations used only cold dark matter (CDM), included only the gravitational force, and usually predicted the dark matter density to go approximately as $1 / \mathrm{r}$ towards the centre of the dark matter halos. Standard parameterizations of these simulated dark matter halos are the Navarro, Frenk and White (NFW)[7] and the Einasto[8] profiles. The latter one is moderately shallower on small spatial scales compared to the NFW profile. N-body simulations showed dark matter profiles that can be both steeper and shallower. Steeper profiles are usually referred to as cuspy profiles. All the dark matter simulations agree on the main halo structure at large distances but the predictive power is limited by the spatial resolution of the simulation, and the shape and density of the profile in the inner part of the halo relies on extrapolation of the simulation prediction. The priority for the CTA DM program is to discover the nature of DM. The publication of limits following non-detection would certainly happen, but in planning the observational strategy, the priority of discovery drives the programme. The possibility of discovery should be considered in the light of model predictions where the minimum goal for searches within the most widely considered models is the thermal cross-section and CTA can probe DM scenarios out of reach for collider searches and direct detection experiments [9]. The principal target for DM observations in CTA is the Galactic halo. This observation will be taken within several degrees of the Galactic centre with the Galactic centre and the most intense diffuse emission regions removed from the analysis. With a cuspy DM profile, observations of 
500 hours in this region provide sensitivities below the thermal cross-section and give a significant chance of discovery in some of the most popular models for WIMPs. Since the DM density in the Galactic halo is far from certain, and considering the irreducible background from cosmic rays and the galactic diffuse emission there, other targets are also proposed for observation. Among these secondary targets, the first to be observed will be dwarf spheroidal satellite galaxies with 100 hours per year proposed. Beyond these two observational targets, alternatives will be considered closer to the actual date of CTA operations. New sky surveys such as the Dark Energy Survey (DES) [10] will extend the knowledge of possible sites of large DM concentrations and a detailed study of the latest data will be made to continuously select the best targets for DM searches in CTA. Among these new possible targets are newly discovered candidate dwarf galaxies and not yet discovered DM clumps (pure DM overdensities without baryonic counterpart and thus deprived of any stellar activity) which could be very promising sources if their locations are identified a priori by their gravitational effects. Beyond the targets proposed for observations in the present Key Science Projects (KSP), the data from the Large Magellanic Cloud (LMC) and from the clusters of Galaxies KSPs will also be used to search for DM. Furthermore, the data from the Galactic plane and extragalactic surveys might give hints of $\gamma$-ray sources which do not have counterparts in multi-wavelength data and which could be pursued as DM targets. The DM Programme is very well suited to being carried out in the Core Programme by the CTA Consortium. The observations require a large amount of time with a significant chance of major discovery but with a clear risk of a null detection. For the Galactic halo the observation time used will also be of great use for astrophysics. In the phase between now and the actual operation of CTA significant evolution in our understanding of DM distributions in the various targets is foreseen and we will adapt our program accordingly. Further detailed work is needed to understand the systematics in the backgrounds especially in the Galactic halo that looks the most promising target up to now.

\section{Galactic halo}

The centre of the Milky Way has in the past been considered as a target for dark matter searches $[13,14]$. More recently, because of the rich field of very high-energy (VHE) $\gamma$-ray astrophysical sources in the region, the searches focus on the Galactic halo excluding the central region of Galactic latitude $b<0.3^{\circ}$. Even excluding the very central region, the total mass of dark matter in the Galactic halo together with its proximity to Earth makes it the most promising source for dark matter searches with CTA. The inconvenience of this target, however, is the fact that being a diffuse source, the integration over the inner halo, while yielding a large signal, gives a very large instrumental background from misidentified charged cosmic rays [15]. Furthermore, there are astrophysical backgrounds from various sources which must be understood, even with the very central region excluded from the analysis. It is believed that the disadvantages of the Galactic halo can be overcome with sufficient experimental effort to control systematic effects in the background subtraction and modeling. The expertise required for this analysis strengthens the case for this programme to be conducted by the CTA Consortium. The Galactic halo observations will be taken with multiple grid pointings with offsets from the Galactic centre position of about $\pm 1.3^{\circ}$ to cover the central $4^{\circ}$ as uniformly as possible. In the Galactic centre KSP a further 300 hours are proposed for astrophysics covering up to latitudes $\pm 10^{\circ}$. These data will also be included in the 

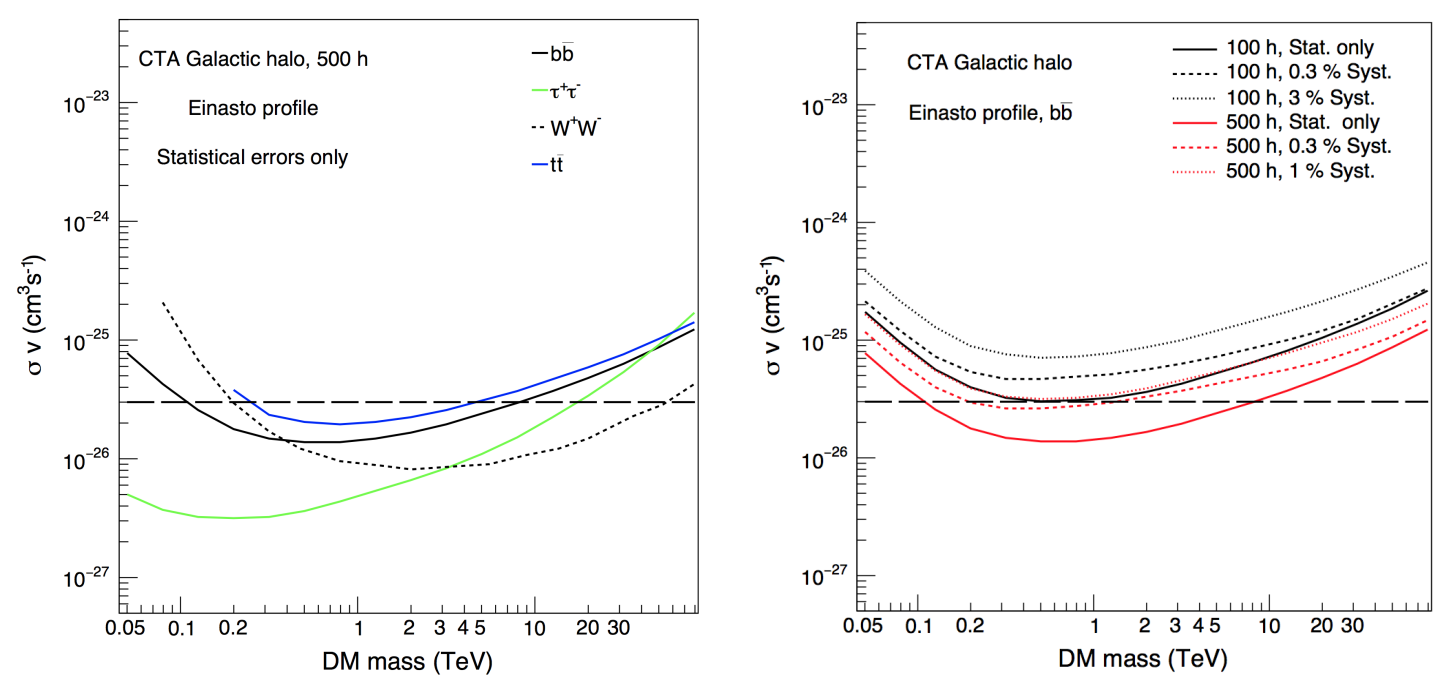

Figure 1: Left panel: CTA sensitivity from observation of the Galactic halo for the Einasto profile and for different annihilation modes as indicated. Only statistical errors were included. Right panel: CTA sensitivity for $b \bar{b}$ annihilation mode for the Einasto profile and for different conditions, black is for 100 hours of observation and red is for 500 hours. The solid lines are the sensitivities only taking into account the statistical errors while the dashed and dotted curves take into account systematics as indicated. The dashed horizontal lines approximate the level of the thermal relic cross-section.

analysis for dark matter to improve the sensitivity for cored dark matter density profiles. Given the major scientific impact of a positive result, we propose that these observations are done in the first three years of CTA operation with high priority. The sensitivity predictions for observations in the Galactic Halo are shown in Figure 1. The left-hand plot shows the sensitivity for different annihilation modes and the right-hand plot for different observation times with a method [16] to include systematic uncertainties on the residual cosmic-ray background as indicated in the caption.

In Figure 2 is shown the CTA sensitivity to a WIMP annihilation signature as a function of WIMP mass for nominal parameters and for the multiple CTA observations. The dashed horizontal line indicates the likely cross-section for a WIMP which is a thermal relic of the Big Bang.

The predictions shown here can be considered optimistic, even when systematics errors are included, as we do not consider the effect of the Galactic diffuse emission as background for DM searches. This will be investigated in detail in a forthcoming publication by the CTA Consortium

\section{Dwarf satellite galaxies}

The dwarf spheroidal galaxies (dSphs) of the Local Group could give a clear and unambiguous detection of dark matter. They are gravitationally bound objects and are believed to contain up to $10^{3}$ times more mass in dark matter than in visible matter, making them widely discussed as potential targets. Being small and distant, many of the dwarf galaxies will appear as near point sources in CTA and hence the nuisance of the instrumental background is much reduced. Although less massive than the Milky Way or the LMC, they are also environments with a favourably low astrophysical $\gamma$-ray background making the unambiguous identification of a dark matter signal 


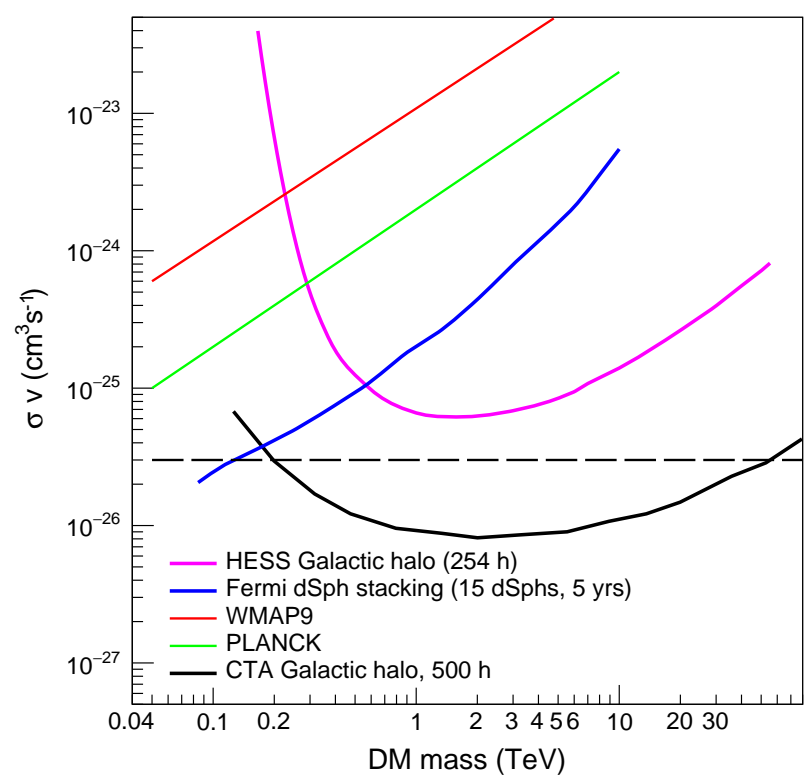

Figure 2: Current best limits on the annihilation cross-section from indirect detection (Fermi-LAT dwarf spheroidal galaxies stacking analysis, $W^{+} W^{-}$channel [11], H.E.S.S. Galactic halo $W^{+} W^{-}$channel [14]) and cosmic microwave background (WMAP and Planck $b \bar{b}$ channel [12]) experiments compared with the projected sensitivity for CTA from observations of the Galactic halo for the Einasto profile, $W^{+} W^{-}$channel. The expectation for CTA is optimistic as it includes only statistical errors. The effect of the Galactic diffuse emission can affect the results by $\sim 50 \%$

easier compared to the Galactic centre or LMC. Neither astrophysical $\gamma$-ray sources (supernova remnants, pulsar wind nebulae,...) nor gas acting as target material for cosmic rays have been observed in these systems.

The search program selects dwarf galaxy targets on the basis of both their J-factors and relative J-factor uncertainties. Due to the larger available sample of spectroscopically measured stars, the classical dwarf galaxies such as Draco, Ursa Minor, Sculptor, and Fornax have significantly smaller uncertainties on the J-factor than the ultrafaint dwarf galaxies [11]. Our knowledge of the dark matter distribution in the so-called classical dSph satellites of the Milky Way is based on dynamical modelling of their internal stellar kinematics [17].

It is reasonable to expect that on a 5-10 year timescale there will be samples of at least 1000 stars for all of the classical dSphs, with Sculptor and Fornax yielding particularly rich samples of $5 \mathrm{~K}$ and $10 \mathrm{~K}$ stars, respectively. These new data will need to be complemented by more advanced modelling in order to constrain their dark matter profiles. Studies are underway to estimate the impact that increased data sets and improved modelling can be expected to have on the ability of CTA to constrain the nature of dark matter The observations of a dwarf spheroidal galaxy will be started in the first year of the Dark Matter Programme. Any hints of dark matter signals or unknown sources would guide the plans for future observations. In the absence of signals, a programme of observation on the most promising $\mathrm{dSph}$ would be taken and the observing strategy is to acquire 100 

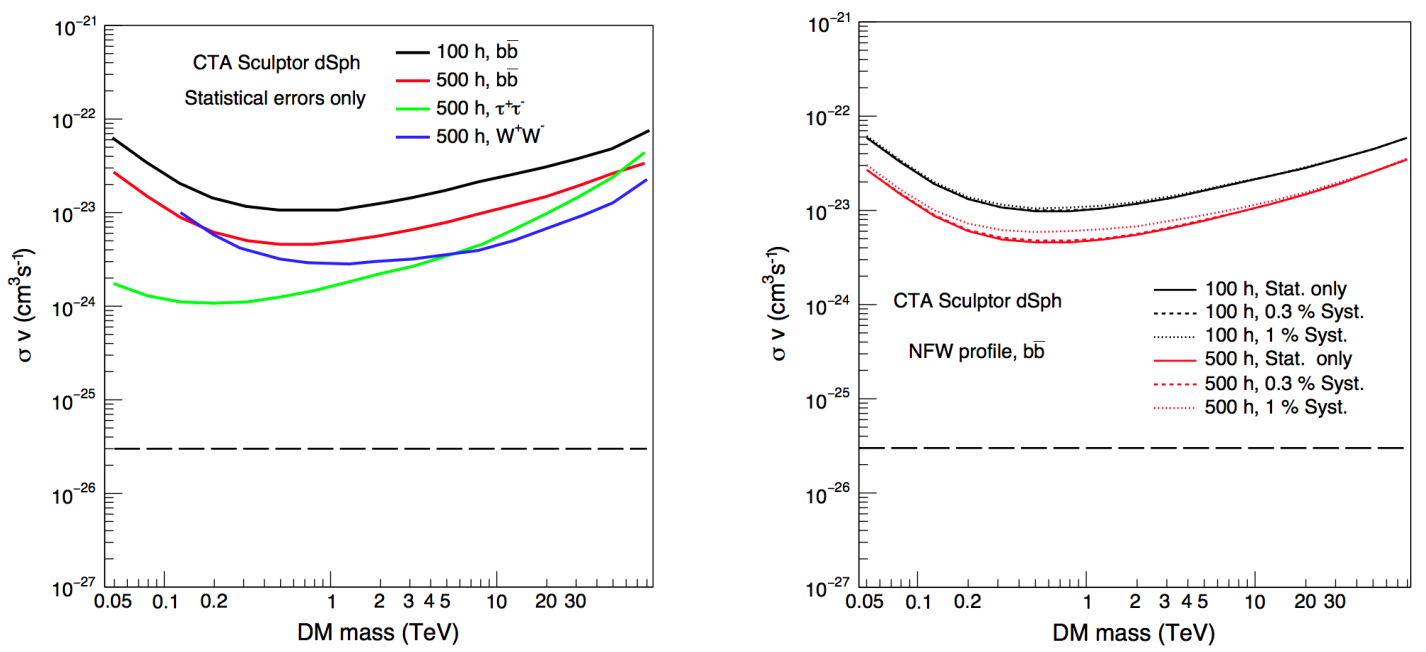

Figure 3: Left panel: Sensitivity for $\langle\sigma v\rangle$ from observation of the classical dwarf galaxy Sculptor for the NFW profile and for different annihilation modes as indicated. Right panel: Sensitivity for $\langle\sigma v\rangle$ for $b \bar{b}$ annihilation modes for different observation times of the same dwarf galaxy. Black line is for $100 \mathrm{~h}$ of observation and red line for $500 \mathrm{~h}$. The solid lines are the sensitivities only taking into statistical errors while dashed and dotted curves take into account systematics as indicated in the figure. The dashed horizontal line shows the thermal cross-section in both figures.

$\mathrm{h}$ of observations per year on the best candidate $\mathrm{dSph}$ at that time. An example of the sensitivity which could be obtained by observations of a classical dwarf galaxy is shown in Figure 3. In making this comparison with Figure 2 it can be seen that the sensitivity is a factor 100 worse for this classical dwarf galaxy; however the effect of systematics is drastically reduced for this small source compared to the extended Galactic Halo, explaining the significant interest in observations of dwarfs. Observations of a classical dSph provide comparable sensitivity to the Galactic centre for the cored dark matter profile and cored profiles in dSphs impact their sensitivity reach by a factor of only a few [5].

\section{Large Magellanic Cloud}

The Large Magellanic Cloud (LMC) is a nearby satellite galaxy at high Galactic latitude and it has the shape of a disk seen nearly face-on. At a distance of only $\sim 50 \mathrm{kpc}$, and with a large dark matter mass of $\sim 10^{10} M_{\odot}$, the LMC has long been recognized as a potentially favorable target for indirect dark matter searches [18]. For the LMC, observations will be taken with several pointings to cover the full galaxy. A total of $340 \mathrm{~h}$ of observations are proposed for both DM and astrophysical motivations. The sensitivity is computed for two benchmark annihilation channels, $b \bar{b}$ and $W^{+} W^{-}$, with the results shown in Figure 4 (left). The curves represent the 95\% confidence level upper limits that would be obtained on the dark matter annihilation cross-section as a function of dark matter particle mass in the case that no emission associated with a dark matter spatial template is detected. The minimum energy considered in the analyses is $200 \mathrm{GeV}$ due to the minimum zenith angles allowed for LMC observations. The strongest sensitivity is achieved for the 

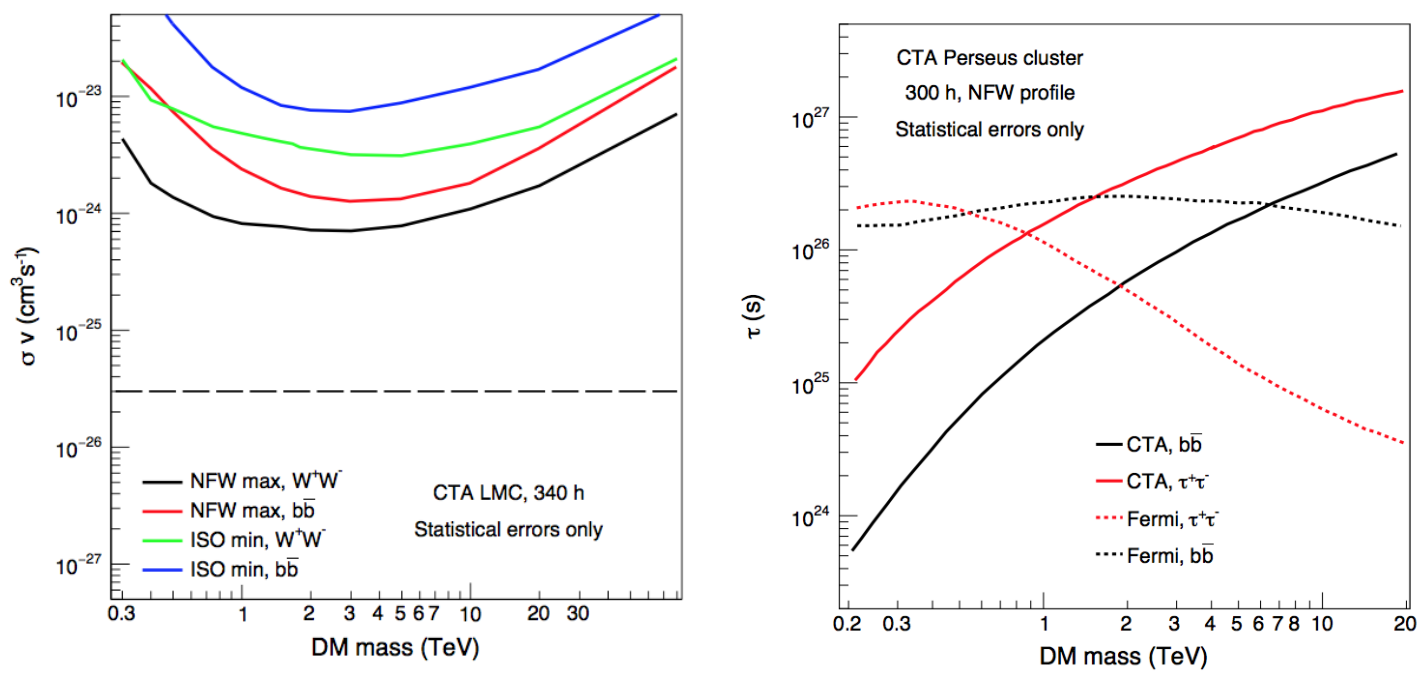

Figure 4: Left panel: CTA sensitivity on $\langle\sigma v\rangle$ from observation of the LMC for 340 hours of observation in the $b \bar{b}$ and $W^{+} W^{-}$annihilation channels for both NFW and isothermal (ISO) dark matter profiles, as shown in the legend. The sensitivities are computed with a $200 \mathrm{GeV}$ energy threshold assuming statistical errors only. Right panel: Expected CTA sensitivity to the dark matter decay lifetime $\tau$ for $300 \mathrm{~h}$ of observation of the Perseus cluster. We assume a dark matter profile as in [21] and adopted the full-likelihood analysis of [20]. The size of the signal integration region ( $0.3^{\circ}$ radius) has been optimised taking into account the expected source extension and the performance for off-axis observations. We assume five off regions. We compare our CTA predictions with the results from the Galactic Halo by Fermi [22].

NFW profile with the maximum rotation curve and maximum allowed density within uncertainties in the inclination angle of the LMC, while the minimum rotation curve with the isothermal profile yields the weakest sensitivity. In the most optimistic case, the expected sensitivity is about a factor of twenty above the thermal relic cross-section. The difference in the testable annihilation crosssection between the extreme cases is a factor of $\sim 10$. The astrophysical backgrounds in the LMC and their uncertainties differ from those in the Galactic centre making it a complementary dark matter search target that offers competitive sensitivity to annihilation signals.

\section{Clusters of galaxies}

Galaxy clusters are a promising target for decaying dark matter (see, for instance [19]). While the signal originating from annihilating dark matter is proportional to the square of the dark matter density, for decaying dark matter the dependence is on the first power. As a consequence, dense dark matter concentrations outshine the astrophysical backgrounds if annihilation is at play, but remain comparatively dim if dark matter is decaying. Decaying dark matter wins instead, generally speaking, when large volumes are considered. Figure 4 (right) shows predictions for the case of the Perseus cluster for $300 \mathrm{~h}$ of observation. We assume a dark matter profile in the cluster as in [21] while adopting the full-likelihood analysis of [20]. We considered an integration radius of $0.3^{\circ}$. As is clear from the figure, CTA can do much better than Fermi [22] at the TeV scale. 


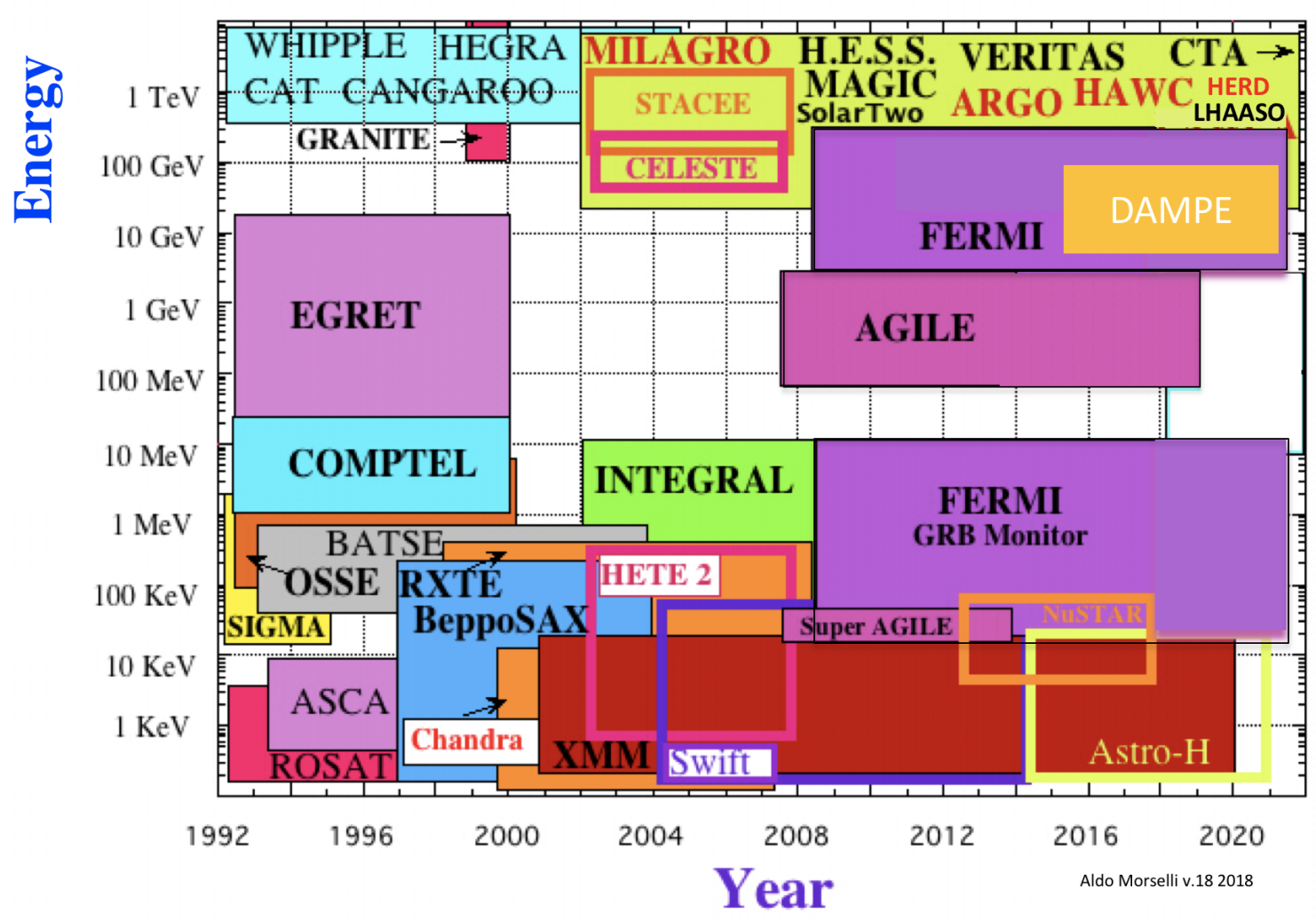

Figure 5: Timeline schedule versus the energy range covered by present and future detectors in $X$ and $\gamma$-ray astrophysics.

\section{The low enery frontier}

In Figure 5 is shown the energy range covered by present and future experiments in $\mathrm{X}$ and $\gamma$-ray astrophysics versus the time of operation. There is an energy range below the Fermi and Agile energies that is not covered by any experiments.

In this energy range a new instrument like Gamma-Light [23] or e-ASTROGAM [24, 25] is needed

The e-ASTROGAM instrument (Figure 6) (right) is based on double-sided Silicon detectors coupled to front-end-electronics capable of acquiring analog information on energy deposition in the range 20-1000 keV with high efficiency and high signal-to-noise. Both Compton events induced by photons in the range $0.3-30 \mathrm{MeV}$ and pair production events in the $30 \mathrm{MeV}-30 \mathrm{GeV}$ range can be detected by the e-ASTROGAM Tracker equipped with a Calorimeter and an Anticoincidence system. Figure 6 (left) shows representative topologies for Compton and pair events. For Compton events, point interactions of the $\gamma$-ray in tracker and calorimeter produce spatially resolved energy deposits, which have to be reconstructed in sequence using the redundant kinematic information from multiple interactions. Once the sequence is established, two sets of information are used for imaging: the total energy and the energy deposit in the first interaction measure the first Compton 

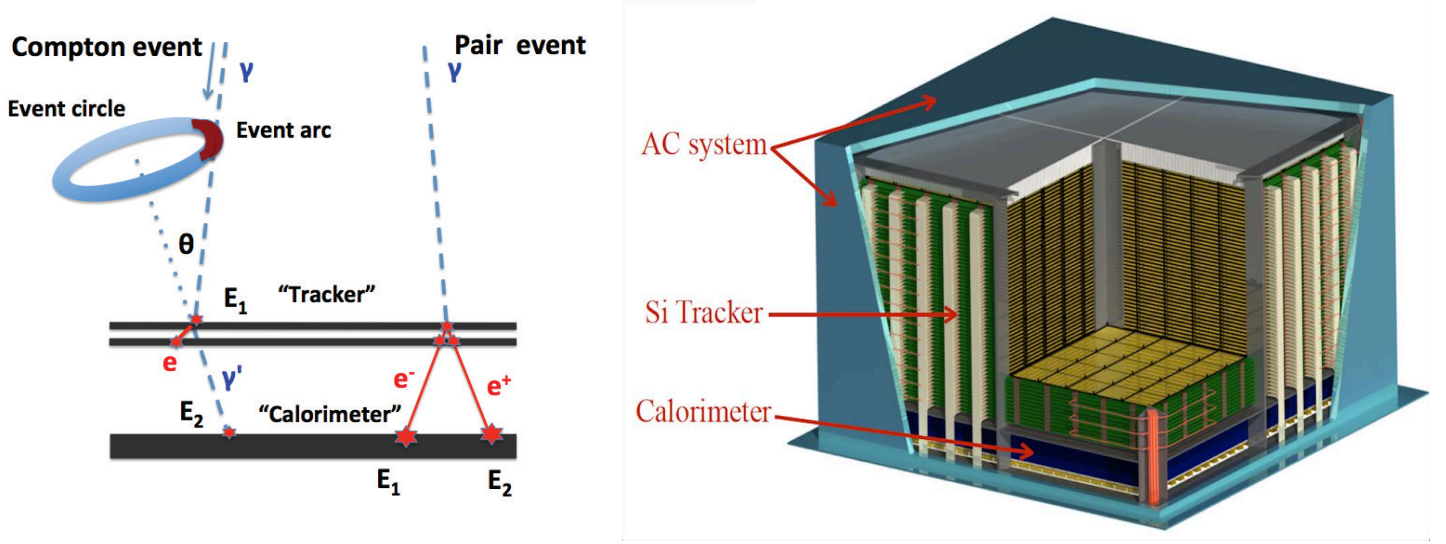

Figure 6: Left: Representative topologies for a Compton event and for a pair event. Photon tracks are shown in pale blue, dashed, and electron and/or positron tracks are in red, solid. Right: Overview of the e-ASTROGAM payload.

scatter angle. The combination with the direction of the scattered photon from the vertices of the first and second interactions generates a ring on the sky containing the source direction. Multiple photons from the same source enable a full deconvolution of the image, using probabilistic techniques. For energetic Compton scatters (above $1 \mathrm{MeV}$ ), measurement of the track of the scattered electron becomes possible, resulting in a reduction of the event ring to an arc, hence further improving event reconstruction. Compton scattering depends on polarization of the incoming photon, hence careful statistical analysis of the photons for a strong (e.g., transient) source yields a measurement of the degree of polarization of its high-energy emission. Pair events produce two main tracks from the electron and positron at small opening angle. Tracking of the initial opening angle and the plane spanned by electron and positron enables direct back-projection of the source. Multiple scattering in the tracker material (or any intervening passive materials) leads to broadening of the tracks and limits the angular resolution at low energies. The nuclear recoil taking up an unmeasured momentum results in a small uncertainty, usually negligible compared to instrumental effects. The energy of the $\gamma$-ray is measured using the calorimeter. Polarization information in the pair domain is given by the azimuthal orientation of the electron-positron plane.

The Point Spread Function of e-ASTROGAM is shown in figure 7 (left), the energy resolution is shown in figure 7 (right) and the sensitivity is shown in figure 8 at $3 \sigma$ for an effective exposure of 1 year of a high Galactic latitude source. The curves for INTEGRAL/JEM-X, IBIS (ISGRI and PICsIT), and SPI are for an effective observation time $T_{\mathrm{obs}}=1 \mathrm{Ms}$. The COMPTEL and EGRET sensitivities are given for the observing time accumulated during the whole duration of the CGRO mission ( $\mathrm{T}_{o b s} \sim 9$ years). The Fermi-LAT sensitivity is for a high Galactic latitude source and $\mathrm{T}_{o b s}$ $=10$ years . For MAGIC, VERITAS (sensitivity of H.E.S.S. is similar) and CTA the sensitivities are given for $\mathrm{T}_{o b s}=50$ hours. For HAWC $T_{\mathrm{obs}}=5 \mathrm{yr}$, for LHAASO $T_{\mathrm{obs}}=1 \mathrm{yr}$, and for HiSCORE $T_{\mathrm{obs}}=1000 \mathrm{~h}$. The very good angular resolution at low energies will help to resolve sources in the galactic center region and to desentangle a possible dark matter contribution ( see figure 9). The core mission science of e-ASTROGAM addresses three major topics of modern astrophysics. 

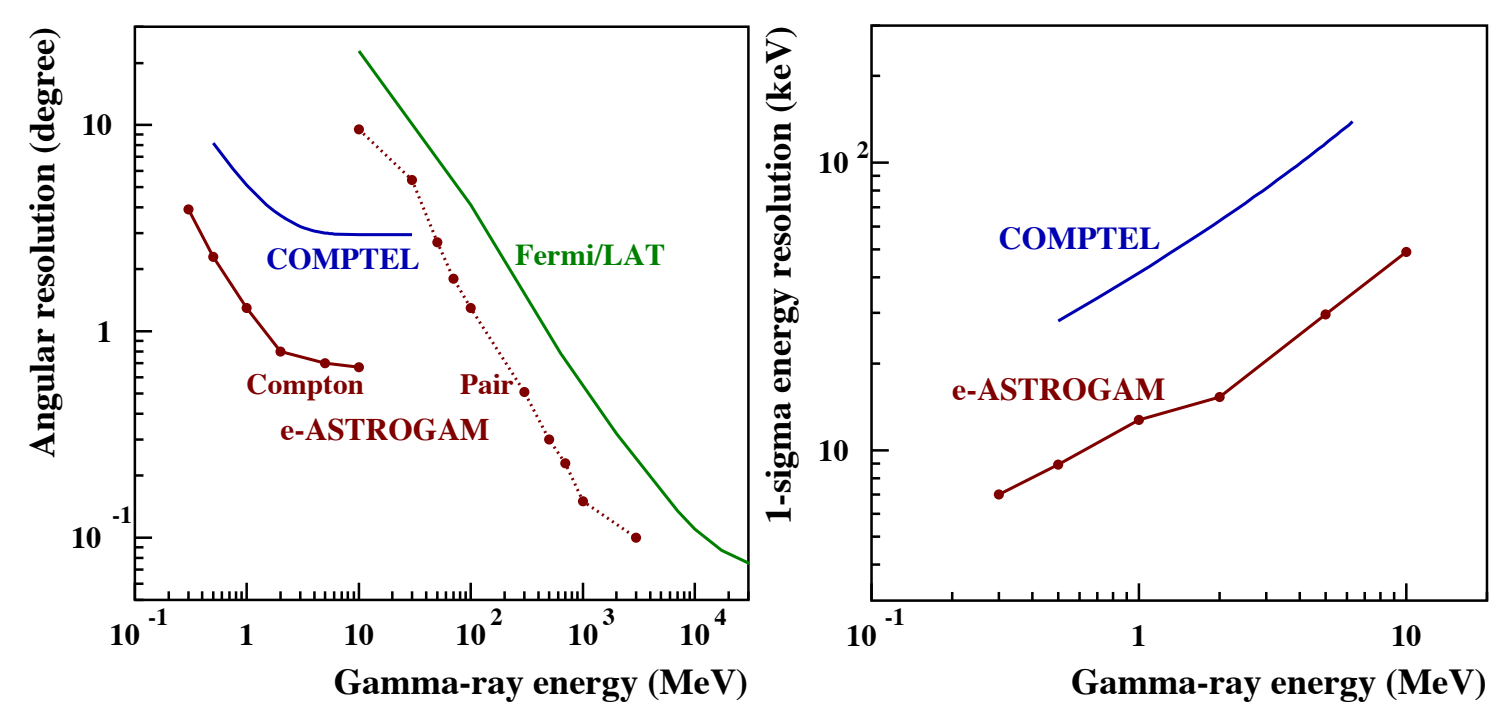

Figure 7: Left: Point Spread Function (PSF, 68\% containment radius) of the e-ASTROGAM $\gamma$-ray detector. For comparison, we show the Fermi-LAT Pass8 PSF and the COMPTEL instrument. In the Compton domain, the performance of e-ASTROGAM and COMPTEL is the FWHM of the angular resolution measure (ARM). Right: $1 \sigma$ energy resolution of COMPTEL and e-ASTROGAM in the Compton domain after event reconstruction and selection on the ARM.

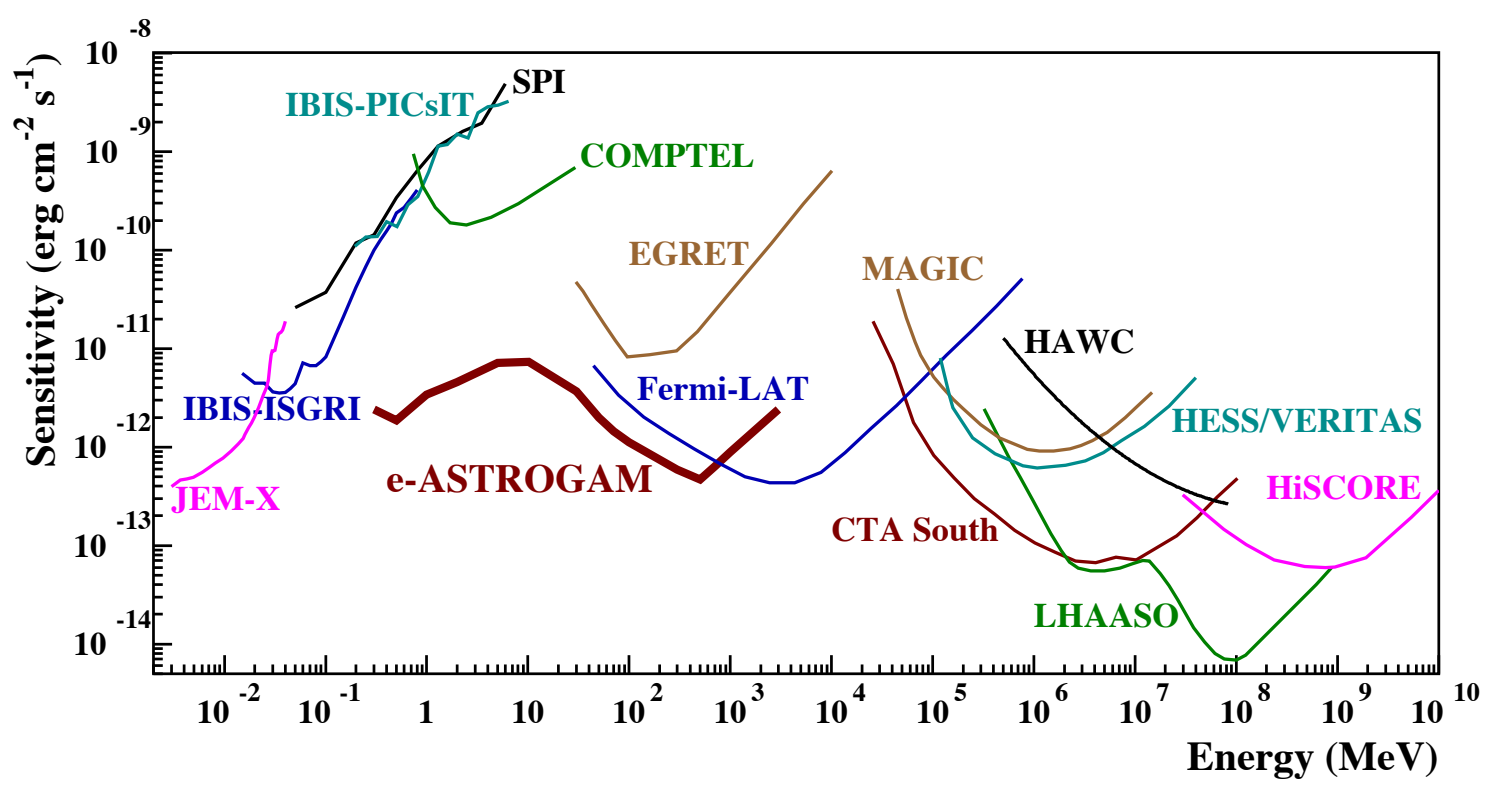

Figure 8: Point source continuum sensitivity of different $X$ and $\gamma$-ray instruments compared with eASTROGAM.

- Processes at the heart of the extreme Universe: prospects for the Astronomy of the 2030s

Observations of relativistic jet and outflow sources (both in our Galaxy and in Active Galactic Nuclei - AGN, briefly) in the X-ray and GeV-TeV energy ranges have shown that the 


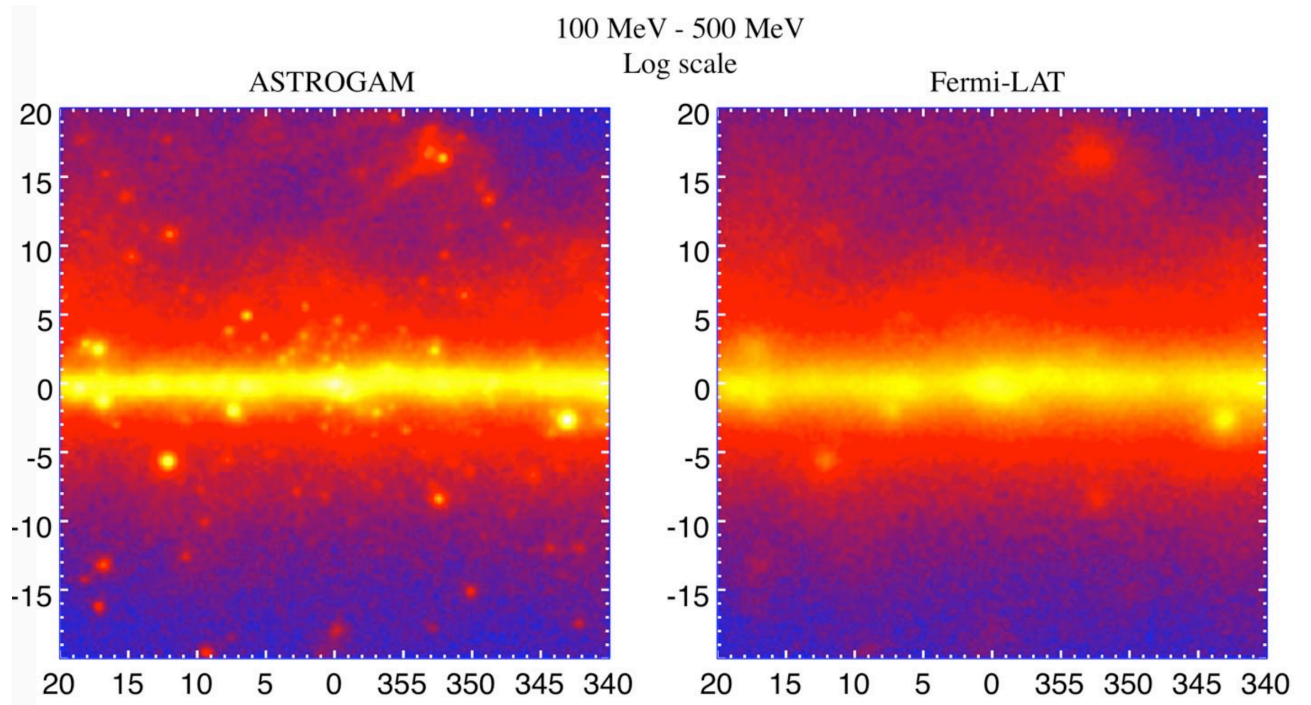

Figure 9: e-ASTROGAM view of the Galactic Center Region in the $100 \mathrm{MeV}-500 \mathrm{MeV}$ energy region compared with the Fermi view.

$\mathrm{MeV}-\mathrm{GeV}$ band holds the key to understand the transition from the low energy continuum to a spectral range shaped by not yet fully understood particle acceleration processes. e-ASTROGAM will: (1) identify the composition (hadronic or leptonic) of the outflows and jets, which strongly influences the environment; (2) identify the physical acceleration processes in these outflows and jets (e.g. diffusive shocks, magnetic field reconnection, plasma effects), that may lead to dramatically different particle energy distributions; (3) clarify the role of the magnetic field in powering ultrarelativistic jets in $\gamma$-ray bursts (GRBs), through time-resolved polarimetry and spectroscopy. In addition, measurements in the eASTROGAM energy band will have a big impact on multimessenger astronomy in the 2030s. After the first joint detection of the GW event GW170817 and the coincident $\gamma$-ray transient GRB 170817A [26] and the ground-breaking insights this first event provided, the future of multimessenger astronomy and the full exploration of its science is of highest priority.

\section{- The origin and impact of high-energy particles on galaxy evolution, from cosmic rays to} antimatter

e-ASTROGAM will resolve the outstanding issue of the origin and propagation of LowEnergy Cosmic Rays (LECRs) affecting star formation. It will measure cosmic-ray diffusion in interstellar clouds and their impact on gas dynamics; it will provide crucial diagnostics about the wind outflows and their feedback on the Galactic environment (e.g., Fermi bubbles, Cygnus cocoon). e-ASTROGAM will have optimal sensitivity and energy resolution to 


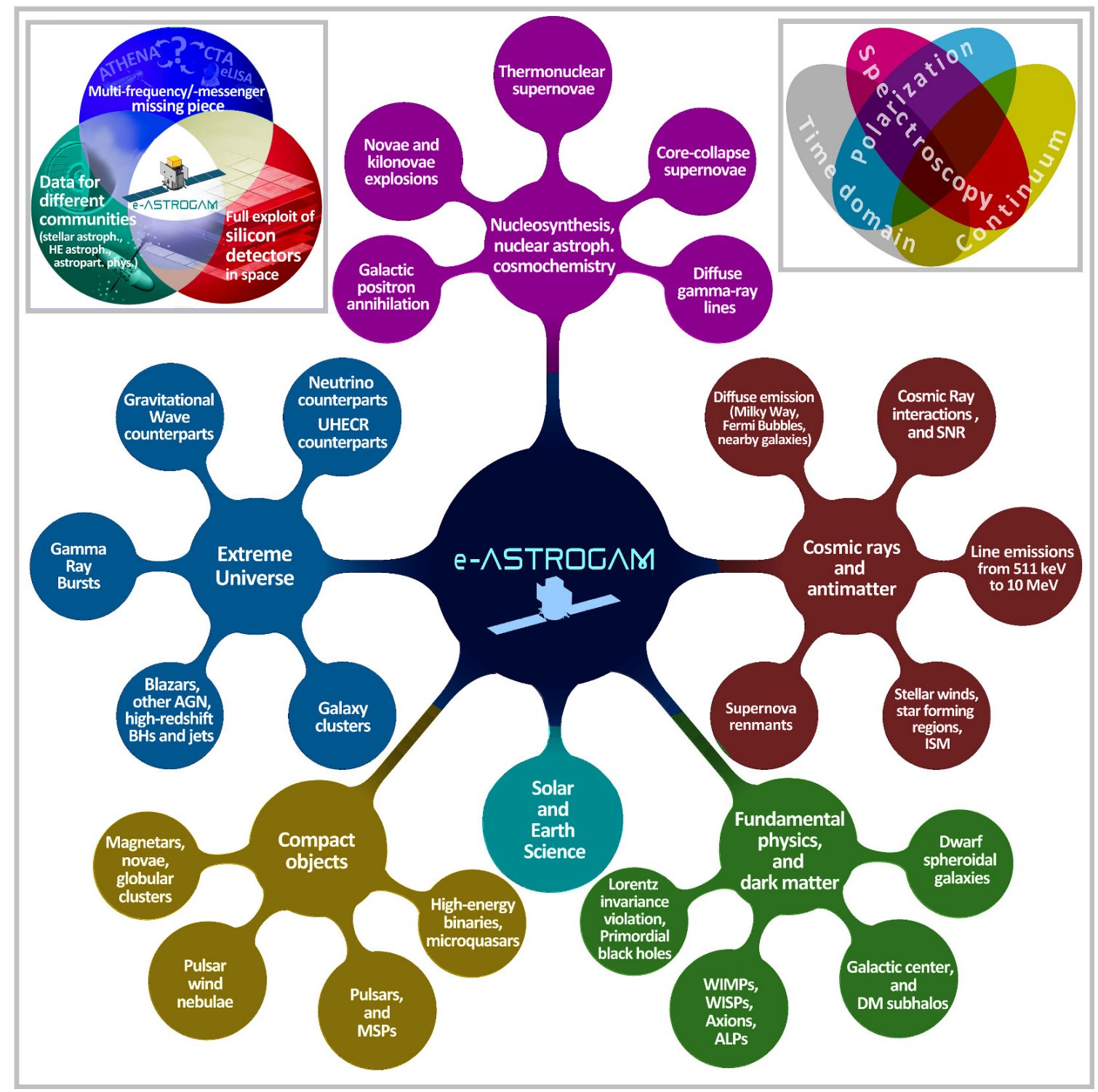

Figure 10: An example of science visualization for e-ASTROGAM illustrating a tentative scheme for science topics and properties of the mission.

detect line emissions in the $\mathrm{keV}-\mathrm{MeV}$ range, and a variety of issues will be resolved, in particular: (1) origin of the $\gamma$-ray and positron excesses toward the Galactic inner regions; (2) determination of the astrophysical sources of the local positron population from a very sensitive observation of pulsars and supernova remnants (SNRs). As a consequence e-ASTROGAM will be able to discriminate the backgrounds to dark matter (DM) signals.

\section{- Nucleosynthesis and the chemical enrichment of our Galaxy}

Thee-ASTROGAM line sensitivity is more than an order of magnitude better than previous instruments. The deep exposure of the Galactic plane region will determine how different isotopes are created in stars and distributed in the interstellar medium; it will also unveil 
the recent history of supernova explosions in the Milky Way. Furthermore, e-ASTROGAM will detect a significant number of Galactic novae and supernovae ( $\mathrm{SNe}$ ) in nearby galaxies, thus addressing fundamental issues in the explosion mechanisms of both core-collapse and thermonuclear SNe. The $\gamma$-ray data will provide a much better understanding of Type Ia SNe which, in turn, will allow to predict their evolution in the past, a pre-requisite for their use as standard candles for precision cosmology.

Beyond the three major "core science" topics of e-ASTROGAM, the list of supplementary, ancillary/bonus, topics can be large and summarized in Fig. 10. Science mapping and visual narration and conceptualization of the e-ASTROGAM instrument properties, simulations, calibrations, data analysis and scientific results, joined to the distribution of high-level data and tools for citizen science exploration, are science communication activities that can be developed in parallel to the main activities for e-ASTROGAM.

\section{Conclusion}

Detection of $\gamma$-rays from the annihilation or decay of dark matter particles is a promising method for identifying dark matter, understanding its intrinsic properties, and mapping its distribution in the universe (in synergy with the experiments at the LHC and in the underground laboratories). In the future it would be extremely important to extend the energy range of experiments at lower energies (compared to the Fermi energies) ( e-ASTROGAM) and higher energies (HAWC, Dampe, HERD, Gamma-400, CTA, LHAASO)

\section{References}

[1] A.Morselli, Constraints on Dark Matter with Gamma-Ray Experiments and Future Observational Strategies, Front. Phys. 5:3. (7pp) 2017, doi: 10.3389/fphy.2017.00003.

[2] Steigman G. et al, Precise relic WIMP abundance and its impact on searches for dark matter annihilation, Phys. Rev. D 86, 2, 023506 (2012).

[3] Acharya B. S. et al, (CTA Consortium), Introducing the CTA concept, Astroparticle Physics 43, 3 (2013)

[4] The Dark Matter Programme of the Cherenkov Telescope Array A.Morselli for the CTA Consortium, PoS(ICRC2017)921 [arXiv:1709.01483]

[5] CTA Consortium, Science with the Cherenkov Telescope Array, World Scientific, https://doi.org/10.1142/10986 [arXiv:1709.07997]

[6] M. Cirelli et al, PPPC 4 DM ID: A Poor Particle Physicist Cookbook for Dark Matter Indirect Detection, JCAP 1103 (2011) 051, erratum: JCAP 1210 (2012) E01 [arXiv: 1012.4515]

[7] Navarro J.F., Frenk C.S. and White S.D. (1996). The Structure of cold dark matter halos. ApJ, 462, 563

[8] Graham A.W., Merritt D., Moore B. et al. (2006). Empirical Models for Dark Matter Halos, Astron.J., 132,2711 
[9] C. Balázs e al., Sensitivity of the Cherenkov Telescope Array to the Detection of a Dark Matter Signal in comparison to Direct Detection and Collider Experiments, Phys. Rev. D 96, 083002 (2017) [arXiv:1706.01505]

[10] Dark Energy Survey Collaboration: T. Abbott et al., The Dark Energy Survey: more than dark energy - an overview, Mon.Not.Roy.Astron.Soc. 460 (2016) no. 2, 1270-1299 [arXiv:1601.00329]

[11] M.Ackermann et al., [Fermi Coll.] Searching for Dark Matter Annihilation from Milky Way Dwarf Spheroidal Galaxies with Six Years of Fermi-LAT Data, PRL 115, 231301 (2015) [arXiv:1503.02641]

[12] Planck Collaboration: P. A. R. Ade et al., Planck 2015 results. XIII. Cosmological parameters A\&A 594, A13 (2016) [arXiv:1502.01589]

[13] Aharonian F. et al. [H.E.S.S. Collaboration] (2006). H.E.S.S. observations of the Galactic Center region and their possible dark matter interpretation. Phys.Rev.Lett., 97, 221102 [arXiv:0610509v2]

[14] H.E.S.S. Collaboration (2017). Search for dark matter annihilations towards the inner Galactic halo from 10 years of observations with H.E.S.S., Phys. Rev. Lett. 117, 111301 [arXiv:1607.08142]

[15] Silverwood H., et al. (2015). A realistic assessment of the CTA sensitivity to dark matter annihilation. JCAP, 1503, 03, 055 [arXiv:1408.4131v2]

[16] Lefranc V. et al. (2015). Prospects for Annihilating Dark Matter in the inner Galactic halo by the Cherenkov Telescope Array. Phys. Rev. D, 91, 12, 122003 [arXiv:1502.05064]

[17] Bonnivard V. et al. (2015). Spherical Jeans analysis for dark matter indirect detection in dwarf spheroidal galaxies - impact of physical parameters and triaxiality. MNRAS, 446, 3002

[18] Tasitsiomi A., Siegal-Gaskins J.M., Olinto A.V. (2004). Gamma-ray and synchrotron emission from neutralino annihilation in the Large Magellanic Cloud. Astropart.Phys., 21, 637

[19] Cirelli M., Moulin E., Panci P. et al. (2012). Gamma ray constraints on Decaying Dark Matter. Phys. Rev., D86, 083506

[20] Aleksić J., Rico J., Martinez M. (2012). Optimized analysis method for indirect dark matter searches with imaging air Cherenkov telescopes. J. Cosmology Astropart. Phys., 10, 032

[21] Sánchez-Conde M.A., Cannoni M., Zandanel F. et al. (2011). Dark matter searches with Cherenkov telescopes: nearby dwarf galaxies or local galaxy clusters?. J. Cosmology Astropart. Phys., 12, 011

[22] Ackermann M. et al. [Fermi Coll.] (2012). Constraints on the Galactic Halo Dark Matter from Fermi-LAT Diffuse Measurements. ApJ, 761, 91 [arXiv:1205.6474]

[23] A.Morselli et al., Nuclear Physics B 239-240 (2013) 193-198 [arXiv:1406.1071]

[24] A. De Angelis et al., The e-ASTROGAM mission (exploring the extreme Universe with gamma rays in the MeV-GeV range, Experimental Astronomy Vol. 44, Issue 1 (58 pp) [arXiv:1611.02232]

[25] A. De Angelis et al., Science with e-ASTROGAM, Journal of High Energy Astrophysics Volume 19, August 2018, Pages 1-106 https://doi.org/10.1016/j.jheap.2018.07.001 [arXiv:1711.01265]

[26] Goldstein, A., Veres, P., Burns, E., et al., 2017, ApJ L, 848, L14 